\title{
The Binding of EGFR to GM1(3) Hosted in Lipid Raft-Like Biomembranes Insighted by Plasmonic Resonance Techniques
}

\author{
Giancarlo Margheri, ${ }^{1}$ Silvana Trigari, ${ }^{1}$ Stefano Sottini, ${ }^{1}$ Riccardo D'Agostino, \\ Tommaso Del Rosso, ${ }^{3}$ and Mario Del Rosso ${ }^{2}$ \\ ${ }^{1}$ Institute for Complex Systems of the National Council of Researches, Via Madonna del Piano 10, 50019 Sesto Fiorentino, Italy \\ ${ }^{2}$ Department of Experimental and Clinical Biomedical Sciences, Section of Experimental Pathology and Oncology, \\ University of Florence, Viale G.B. Morgagni 50, 50134 Florence, Italy \\ ${ }^{3}$ Departamento de Física, Pontificia Universidade Catolica Rio Prédio Cardeal Leme, Sala 652, Rua Marquês de São Vicente 225, \\ 22451-900 Gávea, RJ, Brazil
}

Correspondence should be addressed to Giancarlo Margheri; giancarlo.margheri@cnr.it

Received 4 June 2015; Accepted 14 July 2015

Academic Editor: Banshi D. Gupta

Copyright ( 2015 Giancarlo Margheri et al. This is an open access article distributed under the Creative Commons Attribution License, which permits unrestricted use, distribution, and reproduction in any medium, provided the original work is properly cited.

We exploit $\mathrm{Au} / \mathrm{SiO}_{2}$ plasmonic structures to check the effective binding activity of GM1(3) gangliosides hosted in physiologicallike biomembranes, in presence of the Epidermal Growth Factor Receptor (EGFR). To this aim, we used bilayers that support the propagation of optical surface plasmon modes (plasmonic transducers, PTs) or guided modes (Plasmon Waveguide Resonators, PWRs). First, we measured the binding of EGFR to GM1(3) by using PTs. Indeed, effective interactions were evidenced, but with faint signals that prevented resolving dissociation kinetics. In order to enhance the optical responses, we turned our attention to PWRs. We first refined the design of a previously adopted $\mathrm{Au} / \mathrm{SiO}_{2} \mathrm{PWR}$, finding that the nominal sensitivity is independent on $\mathrm{SiO}_{2}$ thickness but strongly dependent on its residual losses, due typically to a nonoptimal deposition process. We fabricated an improved $\mathrm{Au} / \mathrm{SiO}_{2}$ resonator and tested the predicted signal enhancement by monitoring the binding of EGFR to GM3-enriched biomembranes. The measured signal was $\sim 12$-fold higher than that one measured using a PT, close to the maximum theoretical enhancement. The higher PWR response enabled us to detect the dissociation of EGFR from GM3, and the value of the apparent dissociation constant of the GM3-EGFR complex could be obtained.

\section{Introduction}

Systems mimicking the physiological cell membranes on solid supports (solid supported bilayer lipid membranes, ssBLMs) are excellent templates for studying in vitro their interactions with several biological molecules (ionic pumps, signalling agents, etc....). Many of these molecules are recruited in particular mobile areas of the cell membrane, primarily constituted by sphingomyelin (SM) and cholesterol (C) called lipid rafts [1], where microenvironmental effects on their functional properties can occur, with important pharmacological implications [2]. Among these effectors, gangliosides GM1 and GM3 play important roles in many cell functions [3-7]. In spite of the interest in the fabrication of such structures, there are only a few reports on the formation and functionality of gangliosides-enriched ssBLMs resembling the physiological composition of lipid rafts. In particular, the gangliosides organization in ssBLMs has been locally investigated via sophisticated tools like LangmuirBlodgett equipment, Fluorescence Microscopy or Atomic Force Microscopy [8]. Although these investigations led to significant insights in the effectors nanoscopic organization, their functionality, like the affinity versus specific ligands, could not be easily inferred.

Aiming at insighting the binding affinity between the gangliosides hosted in a physiological-like environment and 
generic ligands, in previous works we demonstrated an alternative simple route to generate GM1(3)-rich SM/C ssBLMs onto silica-gold bilayers by lipid Small Unilamellar Vesicles (SUVs) fusion. Other than effective as ssBLMs growth platforms, $\mathrm{Au} / \mathrm{SiO}_{2}$ bilayers are plasmonic transducers (PTs) that can support propagating surface modes and are exploitable to probe the enrichment of ssBLMs via the binding of the effectors to their specific ligands (CTB and WGA, resp.) by the surface plasmon resonance (SPR) tool $[9,10]$. For the reader's convenience, we first recall in Sections 2 and 3 a brief survey of such work.

Then, in the following paragraphs, we present the results of our recent activity, focused on the measurement of GM1(3) binding to another important molecule, namely, the Epidermal Growth Factor Receptor (EGFR), involved in many important biological functions, primarily the widespread growth of tumours. Indeed, it has been recognized that the presence of gangliosides modulates the functions of EGFR, like its binding to the Epidermal Growth Factor (EGF) [4], process that activates the epidermal cell proliferation, abnormally active in the degenerative step of tumour angiogenesis. Thus, it would be greatly helpful to have a biomodel that resemble the gangliosides-EGFR interaction on a physiological-like basis and can allow a quantitative evaluation of their interaction with a noninvasive and easyto-manage tool. Our SPR-manageable SM/C ssBLMs indeed permitted to observe the EGFR-GM1(3) binding, but with faint responses and poor chances to resolve slow dissociation kinetics. Gaining on our recent work [11], we exploited a modified version of PT with a higher $\mathrm{SiO}_{2}$ thickness [12] that can support guided light modes. In Section 4 we describe a refined design of the device that shows a substantial constancy of the sensitivity versus the $\mathrm{SiO}_{2}$ thickness but a strong sensitivity to its loss, typically caused by a nonoptimized fabrication process, which we had to improve to produce optimized PWRs used to get better binding signals and test the predicted sensitivity. Thereafter, GM3-rich SM/C ssBLMs were sequentially assembled on a PWR sensor surface and the binding of EGFR was detected. We completed the characterization evidencing the dissociation of EGFR from the GM3ssBLMs and estimating the apparent dissociation constant $K_{D}$ of the GM3-EGFR complex.

\section{SPR Tests with CTB and WGA}

The biomimetic raft-like membranes enriched with gangliosides are formed by the fusion of SUVs that occurs when they are in contact with hydrophilic $\mathrm{SiO}_{2}$ interfaces [13]. The fabrication starts with the proper addition of diluted $\mathrm{CHCl}_{3} / \mathrm{MeOH}$ solutions of either GM1 or GM3, in a $\mathrm{CHCl}_{3} / \mathrm{MeOH}$ solution of $\mathrm{SM}$ and $\mathrm{C}$ in $\mathrm{CHCl}_{3}$. The mixtures composition is drawn to produce liposomes GM1(3) $\mathrm{SM}_{0.6-x} \mathrm{C}_{0.4}$, where the suffixes are the molar fractions. After the liquid mixtures have dried, they are hydrated with HEPES buffer solution (HBS), and a yellowish emulsion of multilamellar vesicles (MLVs) is obtained. After sonication, it rearranges in a transparent emulsion of SUVs, with mean radii of $(45 \pm 15) \mathrm{nm}$. The solution is then injected into a PTFE cell, hermetically sealed by the PT, which in this way is exposed to the SUVs. The PTs are SF4 plano-parallel glass slides (Schott glass, refractive index=1.74@633 nm) polished with laser grade finish, covered with $\mathrm{Au} / \mathrm{SiO}_{2}$ films deposited with our high vacuum e-gun assisted evaporation facility. Both $\mathrm{Au}$ and $\mathrm{SiO}_{2}$ layers have equal nominal thicknesses of $50 \mathrm{~nm}$. The uncovered face of the PT is coupled to the hypothenuse of a SF4 roof prism with an index matching oil. The group formed by prism-PT-PTFE cell is hosted into the home-made SPR spectrometer utilized for the optical characterizations of the fusion of the ssBLMs and of the binding reactions. The SPR spectrometer exploits the classical Kretschmann scheme [14] in which the reflectance of a $p$ polarized collimated laser beam of a He-Ne laser (wavelength in vacuum: $633 \mathrm{~nm}$ ) can be measured versus the rotation angle of the PT to obtain the reflectivity versus angle curve (the angular spectrum), or it can be recorded, as a particular case of the so-called sensorgram, at a fixed incidence angle in the highest slope flank of the angular spectrum, typically corresponding to a reflectivity setpoint of 0.3 . A first SPR spectrum is taken after the injection of the SUVs solution before their fusion begins. As the ssBLMs assembly ends, typically after one hour, a second SPR spectrum was recorded after 3 rinses with HBS in order to check the stability of the biomembrane. After the completion of vesicles fusion and verification of the ssBLMs stability, the association between the GM1(3)-enriched ssBLM and ligands was tested by injecting either HBS solutions of the specific ligand for GM1, namely, the $\beta$-subunit of the cholera toxin, CTB, ( $4 \mathrm{nM}$, $8 \mathrm{nM}, 12 \mathrm{nM}, 22 \mathrm{nM}$, and $35 \mathrm{nM})$, the specific ligand for GM3, the wheat germ agglutinin, WGA $(1.2 \mu \mathrm{M}, 2.5 \mu \mathrm{M}, 5 \mu \mathrm{M}$, $10 \mu \mathrm{M}, 20 \mu \mathrm{M}$, and $40 \mu \mathrm{M}$ ) into the PTFE cell, and recording the association sensorgrams for each concentration. After each association phase, we rinsed three times the membrane with pure HBS to eliminate any aspecific binding. Further verifications of the specificity of gangliosides-ligands binding were performed with ssBLM obtained from ganglioside-free SUVs (the control ssBLMs). After the completion of their assembly onto $\mathrm{SiO}_{2}$, they were put in contact with ligands solutions in HBS and the corresponding control sensorgrams were recorded.

The verification of the correct SUVs unrolling, fusion, and ssBLM formation is reported in [9]. The effective presence of gangliosides in ssBLMs is evidenced by well resolved association sensorgrams after the injection of either CTB and WGA solutions in the reaction cell, exemplified by the kinetics of Figure 1, recorded using GM1(3) ${ }_{0.1} \mathrm{SM}_{0.5} \mathrm{C}_{0.4}$ ssBLMs. The control traces (black) reveal no significant ligand capture by the control ssBLMs, suggesting a nice specificity of these systems at least for the recruitment of CTB and WGA. Moreover, the quite different amount of ligands that binds to the ssBLMs loaded with the same nominal molar concentration of ganglioside, circumstance that gives a first indication of the different affinity of the complexes, is also evident. This was confirmed by successive tests performed on $\mathrm{GM} 1(3)_{0.1} \mathrm{SM}_{0.5} \mathrm{C}_{0.4}$ ssBLMs at various concentrations of CTB and WGA. The measured reflectivity variations $S$ versus the molar concentrations of the ligands have been reported in 


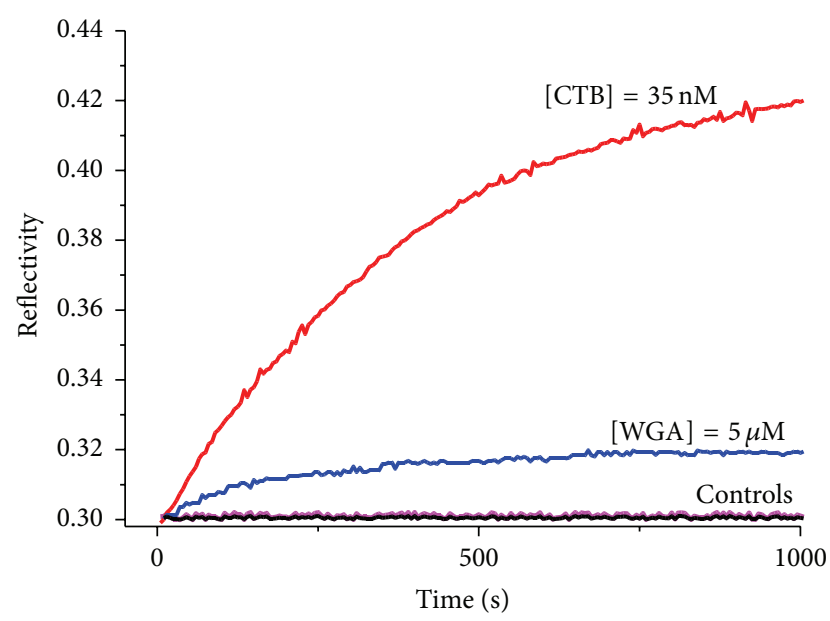

Figure 1: Kinetics of the binding of CTB to GM1 (red) and of WGA to GM3 (blue). The gangliosides were embedded in $\mathrm{GM1}(3)_{0.1} \mathrm{SM}_{0.6} \mathrm{C}_{0.4}$ ssBLMs. The lower traces refer to the interaction of WGA and CTB with ganglioside-free ssBLMs.

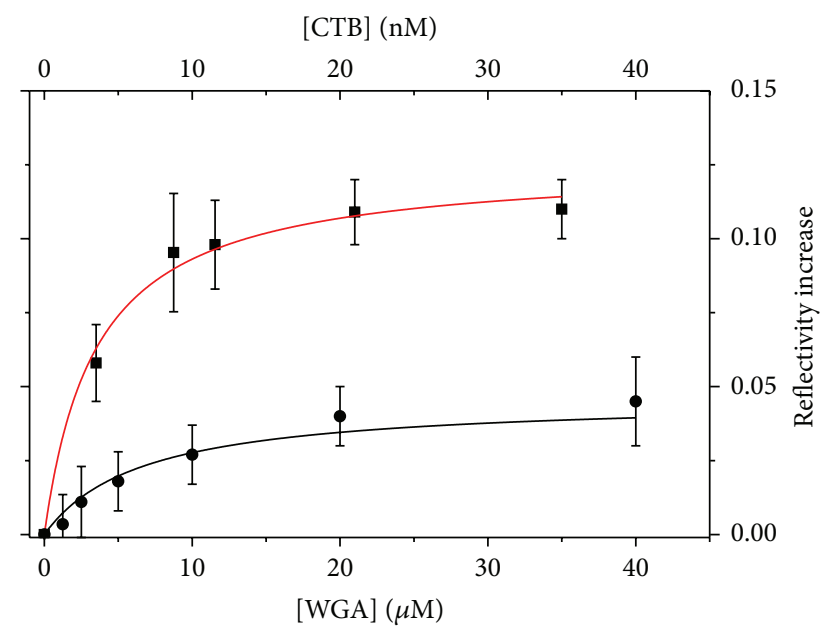

FIGURE 2: Experimental reflectivity increase produced by the binding of CTB to GM1 ( $)$ and of WGA to GM3 (-) together with their best fit curves represented by Langmuir isotherms. The gangliosides were embedded in $\operatorname{GM1}(3)_{0.1} \mathrm{SM}_{0.6} \mathrm{C}_{0.4}$ ssBLMs.

Figure 2 together with their best fit, modeled with Langmuir isotherms, described by the equation:

$$
S=S_{\max } \frac{[A]}{K_{D}+[A]},
$$

where $S_{\max }$ is the reflectivity variation when all the available linking sites are occupied, $[A]$ is the ligand concentration, and $K_{D}$ is the apparent dissociation constant, defined by $K_{D}=k_{\text {diss }} / k_{\text {ass }}$ where $k_{\text {diss }}$ and $k_{\text {ass }}$ are the dissociation rate and the association constant, respectively [15]. The obtained values for the dissociation constants are $K_{D}=(1.5 \pm 0.5) \mathrm{nM}$ and $K_{D}=(9.6 . \pm 0.7) \mu \mathrm{M}$ for CTB-GM1 and WGA-GM3 complexes, respectively.

\section{SPR Tests with EGFR}

Here we report the binding behaviour of EGFR versus GM1(3) by using enriched ssBLM obtained with one-composition mixture GM1(3) ${ }_{0.1} \mathrm{SM}_{0.5} \mathrm{C}_{0.4}$, while a ganglioside-free ssBLM was used as control. The SPR tests were performed by using an EGFR concentration in HBS equal to $35 \mathrm{nM}$.

When the EGFR solution is put into contact with GM1(3)rich ssBLMs, the reflectivity signal $(S)$ increases as shown in Figure 3, demonstrating the effective association of EGFR to both biomembranes, but the control check with gangliosidefree ssBLMs revealed at the same time its nonspecific recruitment, evidenced by the onset of a growing kinetic $S_{\text {control }}$ (Figure 3(a)).

EGFR is a weighty molecule, $\sim 140 \mathrm{kDa}$ molecular weight, and thus its accumulation would be easily detected, provided that its adsorption to the sensor surface is properly efficient. On the contrary, the measured binding signals are quite small, as they reach the maximum reflectivity variation of 0.004 in the case of GM3-rich ssBLM, suggesting that the interaction with the ssBLMs (enriched or ganglioside-free) is intrinsically weak. The differences $S-S_{\text {control }}$ between the kinetic signals (Figure 3(b)), are as well known proportional to the mass of EGFR specifically bound to GM1 or GM3. Although very noisy, they were best fitted with exponential models to give at least a rough comparison between the amounts of bound EGFR on the two gangliosides. The regime best-fit values of the reflectivity variations are $0.13 \%$ and $0.046 \%$ for GM3 and GM1-ssBLMs, respectively, meaning that GM3 binds an amount of EGFR approximately 2.8fold compared to that bound by GM1, in agreement with previous results obtained with immobilized GM1 and GM3 [16]. After the completion of the binding process, we tried to measure the dissociation kinetic by substituting the EGFR solution with pure HBS. Actually, no appreciable dissociation phenomenon was revealed, one possible reason being the low signal to noise ratio that can hide slow decreasing kinetics. In order to better evidence the EGFR-gangliosides interaction, a possibility should be to increase the ligand concentration [15]. However, when dealing with highly purified biosubstances, mainly for costs reasons it is often highly desirable to use the lowest amount of ligand, thus reducing its concentrations in a given volume. Alternatively, high concentrations can be indeed obtained, but in this case complex as-designed microfluidic systems must ensure the proper handling of small volumes of solution. In our case, we decided to maintain the current concentration of receptor $(35 \mathrm{nM})$ and increase the detection sensitivity. To this aim, PWRs seem more appropriate tools than PTs. Increasing the $\mathrm{SiO}_{2}$ thickness above a cut-off value determinates a major concentration of the guided light power in the transparent medium that experiences a much lower propagation loss enhancing the overall resolution of the device [17].

\section{Model and Fabrication of $\mathrm{Au} / \mathrm{SiO}_{2}$ Plasmon Waveguide Resonators (PWRs)}

In previous tests we exploited PWRs to study the affinity of the urokinase Plasminogen Activator Receptor (uPAR) with 


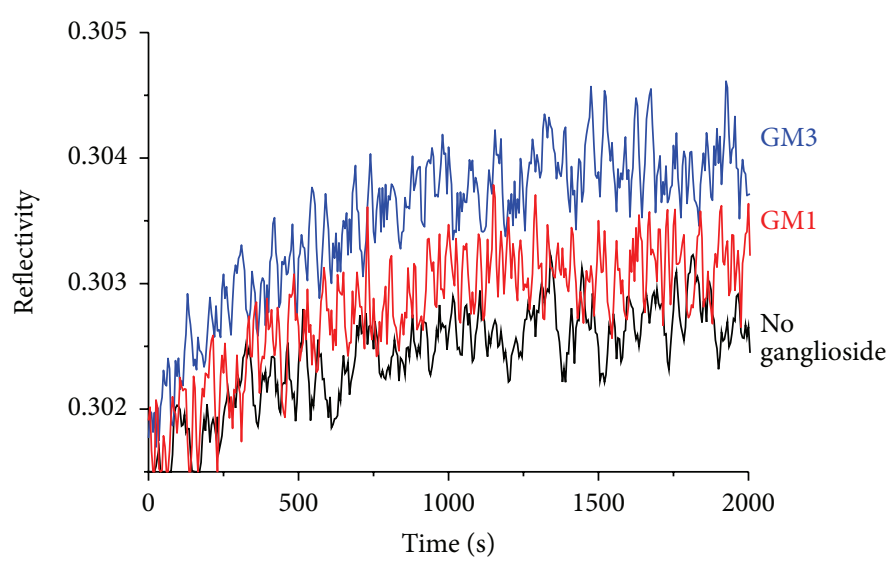

(a)

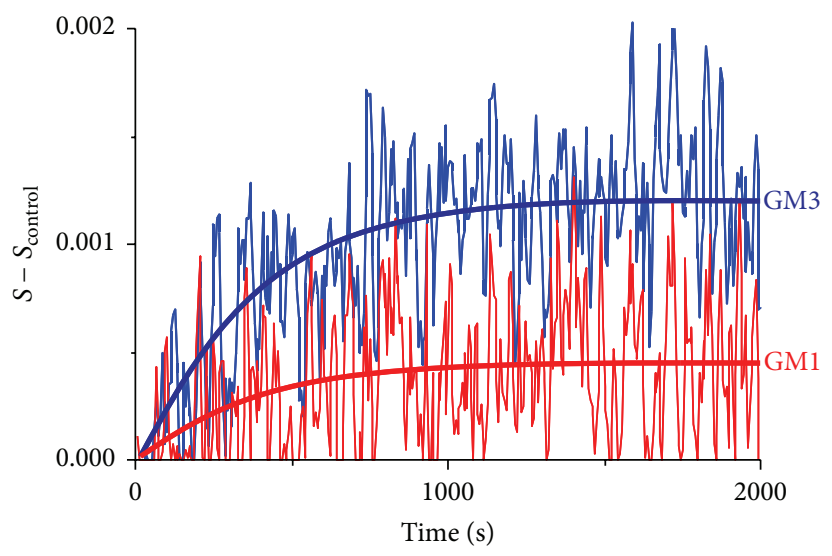

(b)

FIGURE 3: SPR Binding kinetics of EGFR (35 nM in HBS) onto GM1(3) SM $_{0.5} \mathrm{C}_{0.4}$ ssBLMs and ganglioside-free ssBLM as control (a) and after the control subtraction together with their best fit traces.

GM1 and GM3 [8]. Here we refined the design of that device to optimize its sensitivity and to issue possible critical aspects that were not fully elucidated at that time.

At first we modeled the PWR considering an $\mathrm{Au} / \mathrm{SiO}_{2}$ bilayer where the dielectric constant of $\mathrm{Au}$ is fixed and equal to $-11+i$, the $\mathrm{SiO}_{2}$ has a fixed dielectric constant equal to 2.13, and its external surface is in contact with the HBS (dielectric constant $=1.774$ ). The external surface is covered with a layer of thickness $s=5.4 \mathrm{~nm}$ and refractive index equal to 1.5 that simulates the presence of a ssBLM (9). The PWR is excited as seen in Section 2 through a SF4 prism with a collimated beam in either $s$ (TE) or $p$ (TM) polarizations. The incidence angle is chosen in correspondence to a reflectivity setpoint of 0.3 in the negative slope flank of the angular spectrum. Starting from the cut-off thickness of the $\mathrm{SiO}_{2}$ layer corresponding to the $\mathrm{TE}_{0}$ mode, we first identify the gold thickness that minimizes the reflectivity, thus obtaining the optimal optical coupling. Then, we evaluated the sensitivity $(d R / d s)_{s=5.4 \mathrm{~nm}}$ that we define as the reflectivity $(R)$ change per unitary increase of the layer thickness $s$. The procedure is iterated by varying the thickness of $\mathrm{SiO}_{2}$ from the cut-off value of the $\mathrm{TE}_{0}$ mode to that one of $\mathrm{TE}_{1}$ mode. The performances of the $\mathrm{TE}_{0}$-operating PWR are compared to those calculated for the $\mathrm{TM}_{0}$ mode. The modeling of the multilayer has been carried out with WINSPALL software facility. The cut-off thicknesses for $\mathrm{TE}_{0}$ and $\mathrm{TE}_{1}$ modes resulted 270 and $770 \mathrm{~nm}$, respectively, while the optimal gold thickness was found to be $44 \mathrm{~nm}$, corresponding to a residual reflectivity equal to 0.003 . Then, following the iteration procedure, we calculated the sensitivity of the $\mathrm{TE}_{0}$-operating PWR. The results, reported in Figure 4, show that $d R / d s$ maintains quite independent from the $\mathrm{SiO}_{2}$ thickness $\left(\sim 27 \% \mathrm{~nm}^{-1}\right)$ in between the $\mathrm{TE}_{0}$ and $\mathrm{TE}_{1}$ cut-off values that means that the fabrication tolerances on the thickness of the dielectric layer are a minor concern.

When operating at the $\mathrm{TM}_{0}$ mode (cut-off $\mathrm{SiO}_{2}$ thickness = $390 \mathrm{~nm}$ ), the PWR exhibits a significantly lower sensitivity $\left(7 \% \mathrm{~nm}^{-1}\right)$. Both TE and TM sensitivities are however higher than those, $1.8 \% \mathrm{~nm}^{-1}$, exhibited by the PTs optimized for

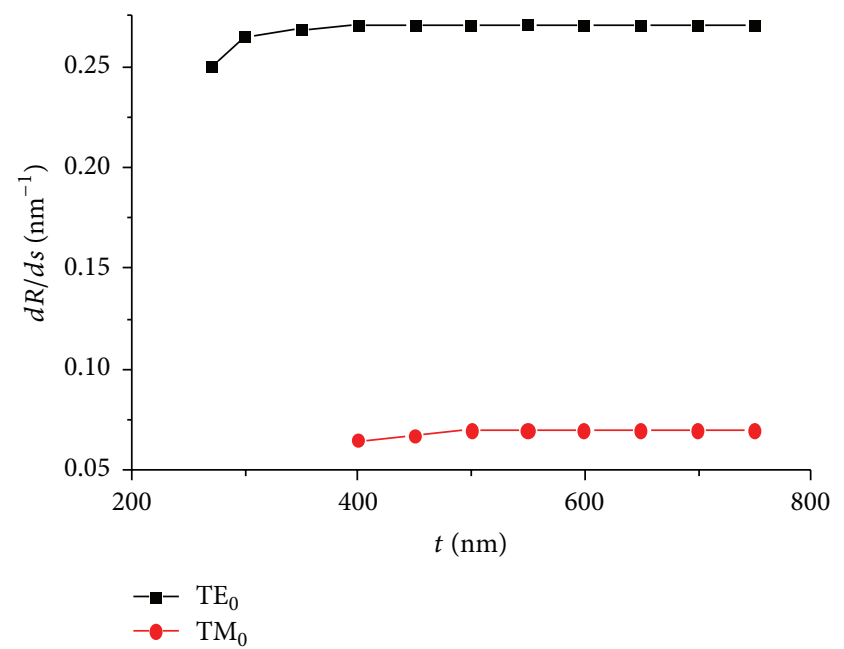

Figure 4: Sensitivity of $\mathrm{Au} / \mathrm{SiO}_{2}$ PWR versus the quartz thickness for TE and TM polarizations. The higher thickness reported $(770 \mathrm{~nm})$ is the cut-off value for $\mathrm{TE}_{1}$-mode.

the experiments. Considering $\mathrm{a} \mathrm{SiO}_{2}$ thickness of $450 \mathrm{~nm}$, the enhancement is evidenced in Figure 5, where the calculated reflectivity changes for the $\mathrm{TE}_{0}$-operating PWR (black arrow) and for the PT (red arrow) are compared and the thickness $s$ increases from its initial value, $5.4 \mathrm{~nm}$, to $6.4 \mathrm{~nm}$. In the case of the PWR spectrum, this $1 \mathrm{~nm}$ increase is sufficient to produce a reflectivity jump-on of the signal in a nonlinear region with lower sensitivity. For this reason, a further layer growth beyond $1 \mathrm{~nm}$ would not be detected with a sufficient resolution.

Concerning the fabrication of PWR, in our previous work [8], we fitted the experimental PWR spectrum only by imposing an imaginary part of quartz dielectric constant of $8 \times 10^{-4}$, responsible for the strong increase of the minimum reflectivity accompanied by a reduction in the sensitivity to about one-half its design value. The presence 


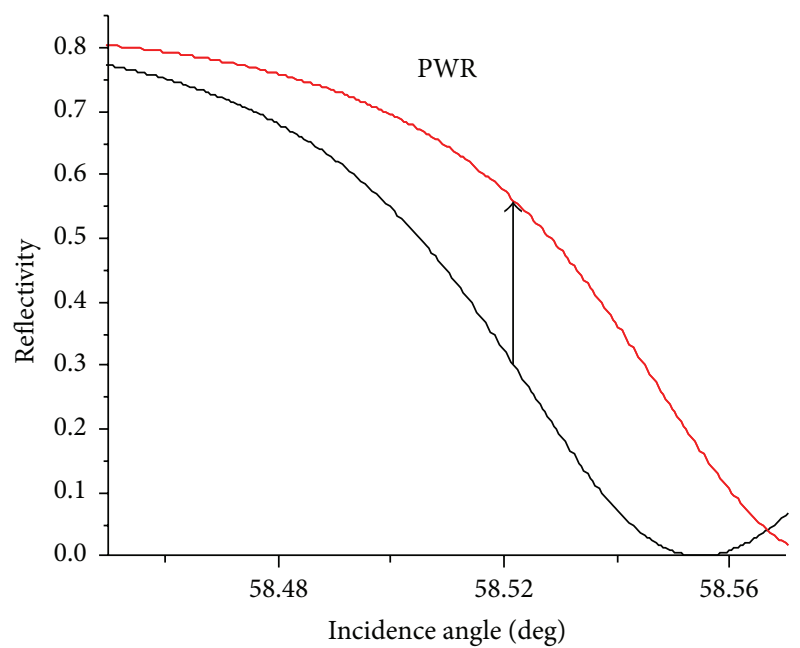

(a)

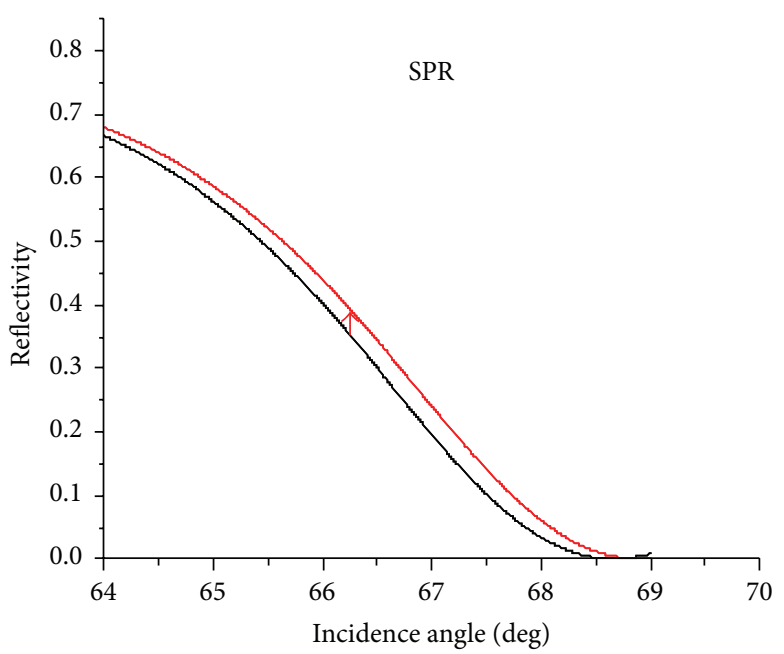

(b)

FIGURE 5: Modeling of the reflectivity increase of a ssBLM-coated PWR upon the accumulation of 1 nm thick ligand layer, provoking a right shift of the initial spectra (black) to their final positions (red). The parameters of the multilayers are given in the text. The external medium is HBS.

of a nonnegligible loss mechanism in the dielectric may be ascribed to the presence of absorbing impurities. Indeed, during the growing process, if the evaporation rate is not proper, clusters of $\mathrm{Si}$ can segregate from the condensing oxides, becoming nucleation centers for the formation of Si nanoparticles [18] that can reduce the transparency of the silica layer and, accordingly, the overall sensitivity. As a consequence, the preadjustment of the evaporation rate comes out to be a necessary prerequisite for the production of a high-quality $\mathrm{SiO}_{2}$ layer. Recently, this precalibration was accomplished by evaporating at various rates (from 0.3 to $2 \mathrm{~nm} \mathrm{~s}^{-1}$ ) $\mathrm{SiO}_{2}$ layers with equal $450 \mathrm{~nm}$ nominal thicknesses onto $44 \mathrm{~nm}$ thick gold layers and measuring the angular spectra of the $\mathrm{TE}_{0}$-mode with air as substrate. The quality of $\mathrm{SiO}_{2}$ layer was considered satisfactory when the PWR spectrum had a residual reflectivity of $\sim 0.05$, to be compared with the theoretical minimum (0.003). This discrepancy is attributable to the tolerances in the gold layer fabrication (some nanometers). We found that this condition was achieved with an evaporation rate of $\sim 1 \mathrm{~nm} \mathrm{~s}^{-1}$ at a residual pressure of $5 \times 10^{-6}$ Torr.

Two PWR samples (measure and control) were fabricated with the assessed rate of evaporation. Two PTs (nominally equal to those of Sections 2 and 3) were also fabricated with the same evaporation conditions to compare the binding signals with those obtained with PWRs.

One PWR sample was put into contact with HBS in the reaction cell and its angular spectrum was recorded. Surprisingly, we found a nonnegligible instability of the spectrum that suffers an angular time shift that we attribute to adjustment phenomena of the outer surface of the $\mathrm{SiO}_{2}$ matrix, readily resolved thanks to the high sensitivity of the PWR [17]. It took $\sim 2$ hours until reflectivity variations smaller than 0.001 in the left flexpoint of the spectrum were stably recorded.
Once the spectrum reached a steady position, its best fit permitted calculating the refractive index and thickness of the $\mathrm{Au}$ and $\mathrm{SiO}_{2}$ layers. The steady spectrum together with its best fit trace is shown in Figure 6. The thickness of the gold layer results $49 \mathrm{~nm}$, with its dielectric constant $-11.0+1.3 i$, while the $\mathrm{SiO}_{2}$ thickness and the dielectric constant were $455 \mathrm{~nm}$ and 2.132 , respectively, in good agreement with the design ones. As anticipated, the residual difference between the measured minimum reflectivity $(0.056)$ and the theoretical one $(0.003)$ can be justified by the mismatch between the experimental and theoretical gold thicknesses. The calculated sensitivity is $22 \% \mathrm{~nm}^{-1}$ instead of the maximum achievable $\left(27 \% \mathrm{~nm}^{-1}\right)$, but still 12.2 times higher than that achievable with an optimized PT.

\section{Comparative Tests Performed with $\mathrm{Au} / \mathrm{SiO}_{2}$ PWRs and PTs}

The expected improvement of the reflectivity variation was checked by performing binding tests between GM3 and EGFR described in Section 2.

A GM3-ssBLM was let to assemble onto the PWR outer surface from a $\mathrm{GM}_{0.1} \mathrm{SM}_{0.5} \mathrm{C}_{0.4}$ emulsion of SUVs. The fusion of the SUVs onto the $\mathrm{SiO}_{2}$ was monitored by recording the PWR spectra at different times and checking their conformal right-shift as monitor of the homogeneity of the ssBLM. After about $30 \mathrm{~min}$, the resonance angle reached a stable value, $58.83^{\circ}$, whose variation with respect to the initial value, $58.7^{\circ}$, is consistent with the effective formation of an adlayer $5.1 \mathrm{~nm}$ thick with a refractive index 1.56, close to the values previously measured in [9]. Subsequently, the $35 \mathrm{nM}$ solution of EGFR in HBS was inserted in the reaction cell and the EGFR-GM3 binding kinetic was recorded. As aspecific 


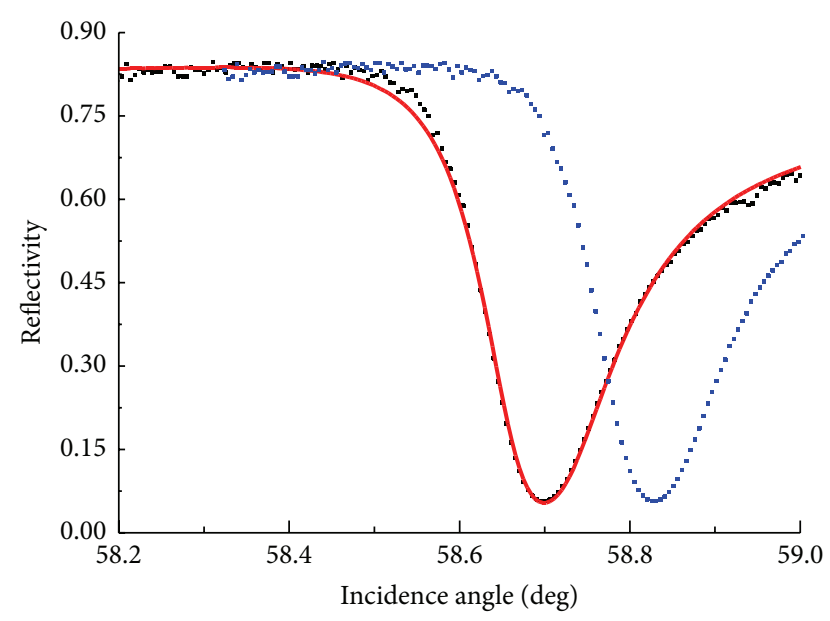

FIgURE 6: The experimental angular spectrum ( $\mathbf{\square})$ and best fit curve (red line) of the fabricated $\mathrm{Au} / \mathrm{SiO}_{2} \mathrm{PWR}$. The external medium is HBS. Blue dots: the angular spectrum after the assembly of GM3ssBLM.

binding was found in SPR tests (Section 2), control kinetics (not reported) were also recorded after having formed a ganglioside-free ssBLM onto the other fabricated PWR and contacted with the EGFR solution. We found that the amount of EGFR present on the control ssBLM was only about $8 \%$ of that one measured in presence of GM3 recorded after having formed a ganglioside-free ssBLM onto the other fabricated PWR and contacted with the EGFR solution. We found that the amount of EGFR present on the control ssBLM was only about $8 \%$ of that one measured in presence of GM3.

A similar procedure was repeated to record the binding and control kinetics with PTs.

The growing kinetics obtained with both PT and PWR after the rinsing and the subtraction of the control kinetics are reported in Figure 7 (reflectivity changes, $\Delta R$ ). Their best fit allowed us to calculate the association rates [15] that were quite close $k_{\text {on }}=(1.15 \pm 0.40) \times 10^{-3} \mathrm{~s}^{-1}$ and $k_{\text {on }}=$ $(1.4 \pm 0.2) \times 10^{-3} \mathrm{~s}^{-1}$ for the PT and PWR, respectively, and whose weighted average results $k_{\text {on }}=(1.3 \pm 0.17) \times 10^{-3} \mathrm{~s}^{-1}$ that we consider as representative of the binding rate of EGFR on ssBLMs grown onto virtually equal $\mathrm{SiO}_{2}$ interfaces. The growing kinetic recorded with PWR has an amplitude approximately 12 -fold higher than that one obtained with PT, in nice agreement with the calculated enhancement (12.2).

After $1800 \mathrm{~s}$ incubation, we substituted the EGFR solution with pure HBS. While with PT we could not reveal any dissociation phenomenon, it could be clearly evidenced with PWR by the occurrence of a clear decreasing kinetic [Figure 8], whose best fit permits calculating the dissociation rate $k_{\text {diss }}=(4.6 \pm 0.4) \times 10^{-3} \mathrm{~s}^{-1}$, mainly attributable to the dissociation of the receptor from GM3. Considering the relationship between the fast association constant $k_{\text {ass }}$ and the association rate $k_{\text {on }}=k_{\text {diss }}+k_{\text {ass }}$ [EGFR], [15], where [EGFR] is the molar concentration of EGFR, we find $k_{\text {ass }}=(3.7 \pm$ $0.5) \times 10^{4}(\mathrm{Ms})^{-1}$. Finally, the value of $K_{D}=(0.12 \pm 0.036) \mu \mathrm{M}$ for the dissociation constant of GM3-EGFR complex can be calculated as the ratio $k_{\text {diss }} / k_{\text {ass }}$.

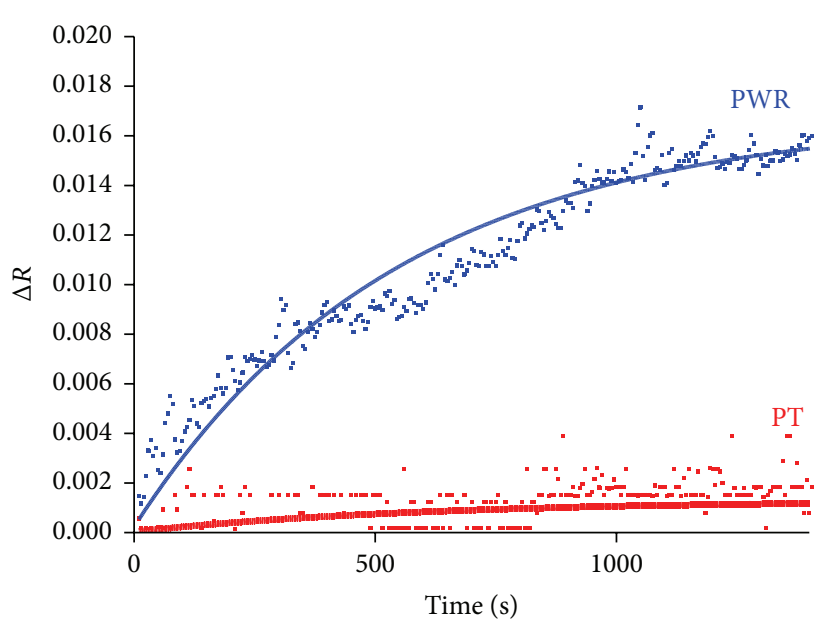

Figure 7: Binding kinetics of EGFR onto a GM3-SM/C ssBLM biomembrane grown onto a PWR and onto a PT after the controls subtraction.

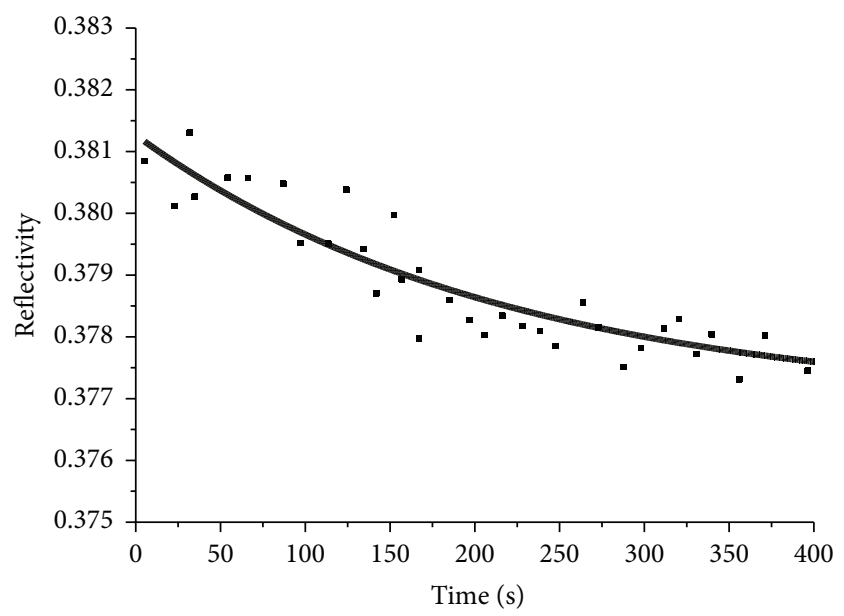

FIgUre 8: Dissociation kinetic (dots) of EGFR from a $\mathrm{GM}_{0.1} \mathrm{SM}_{0.5} \mathrm{C}_{0.4}$ ssBLM recorded using the $\mathrm{Au} / \mathrm{SiO}_{2} \mathrm{PWR}$ described in Section 5, together with its best fit (continuous line).

We repeated three more times the described procedure to check the reproducibility of $K_{D}$. Before each test, we removed in situ the existing ssBLM by rinsing the PWR surface with piranha solution and making sure of the stability of the PWR spectrum in HBS before the new SUVs injection. We obtained a satisfactory reproducibility of the measurements, as their average value resulted $K_{D}=(0.13 \pm 0.046) \mu \mathrm{M}$.

The few literatures present on this subject make a comparison with other data difficult, which furthermore refer to hosting environments and/or molecules which, though having some analogy with ours, exhibit however also significant differences. For instance, our value is more than one order of magnitude lower than the findings of [19], where a guess value of $2 \mu \mathrm{M}$ for the binding affinity between GM3 and the extracellular domain (ECD) of EGFR is reported, 
while in [20] the affinity of EGFR for immobilized derivative lyso-GM3 and its mimetics was measured as $1 \mu \mathrm{M}$, 8-fold the value found in this work.

\section{Conclusions}

We performed SPR analysis with PTs to evidence the EGF receptor effective binding with both GM1 and GM3 that resulted more pronounced in the case of EGFR-GM3 interaction, in agreement with previous findings. However, the faint signals completely prevented the detection of the EGFR dissociation. To get rid of these drawback without increasing the ligand concentration, we exploited the higher sensitivity of PWRs, previously used to study uPAR-gangliosides interaction. Here we presented a refined design of the PWR device, whose most significant feature is that the maximum theoretical sensitivity $\left(27 \% \mathrm{~nm}^{-1}\right)$ is quite independent on the $\mathrm{SiO}_{2}$ thickness, suggesting that the control on its growth during the fabrication can be considered as a minor concern. Rather, we found a strong dependence of the sensitivity on the imaginary part of the dielectric constant of the nominally transparent $\mathrm{SiO}_{2}$ layer. For instance, the occurrence of an imaginary part as small as $8 \times 10^{-4}$ is sufficient to reduce the sensitivity to approximately one-half its theoretical maximum. This finding suggests that a preadjustment of the deposition parameters of $\mathrm{SiO}_{2}$, and in particular its deposition rate, is mandatory to fabricate optimized layers and maintain a high sensitivity. Taking advantage of this result, we fabricated improved samples of $\mathrm{Au} / \mathrm{SiO}_{2} \mathrm{PWR}$ to check the EGFR-GM3 interaction with higher signals and measure the PWR sensitivity at the same time. We found that the signal amplitude with PWR is $\sim 12$-fold that one found with PTs, enhancement not far from the theoretical maximum. The higher resolution of PWR allowed us to put in evidence the dissociation of EGFR from the biomembrane. Thanks to its higher sensitivity, a clear decreasing kinetic could be recorded, and a first evaluation of the apparent dissociation constant was calculated to be $K_{D}=(0.13 \pm$ $0.046) \mu \mathrm{M}$, interestingly compatible with other findings on similar systems. Thus, our results indicate that PWRs can be fruitfully used for more extended, in depth investigations of the interaction of EGFR and gangliosides hosted in raft-like physiological mimicking ssBLM. However, as a final remark, it is worthy noticing that the higher PWR sensitivity versus PTs is gained at expense of (1) a more complex fabrication; (2) need of prolonged prestabilization of the dielectric matrixliquid interface; (3) a reduction of the sensor dynamic that hinders the detection of adsorbed layer thicker than $\sim 2 \mathrm{~nm}$. These features suggest that the use of a PWR as affinity probe must be carefully pondered and adopted when the more flexible and easier to fabricate PTs do not offer chances to evidence affinity phenomena with the appropriate resolution.

\section{Conflict of Interests}

The authors declare that there is no conflict of interests regarding the publication of this paper.

\section{Acknowledgments}

This work was supported by grants of the Ente Cassa di Risparmio di Firenze (ECRF), Istituto Toscano Tumori (ITT), Associazione Italiana Ricerca sul Cancro (AIRC Grant no. IG 2013 N. 14266); Brazilian FAPERJ and fundings from Project UNIFI-4 MELOTAC.

\section{References}

[1] N. M. Hooper, "Membrane biology: do glycolipid microdomains really exist?" Current Biology, vol. 8, no. 4, pp. R114-R116, 1998.

[2] B. Chini and M. Parenti, "G-protein coupled receptors in lipid rafts and caveolae: how, when and why do they go there?" Journal of Molecular Endocrinology, vol. 32, no. 2, pp. 325-338, 2004.

[3] I. Mocchetti, "Exogenous gangliosides, neuronal plasticity and repair, and the neurotrophins," Cellular and Molecular Life Sciences, vol. 62, no. 19-20, pp. 2283-2294, 2005.

[4] A. Rebbaa, J. Hurh, H. Yamamoto, D. S. Kersey, and E. G. Bremer, "Ganglioside GM3 inhibition of EGF receptor mediated signal transduction," Glycobiology, vol. 6, no. 4, pp. 399-406, 1996.

[5] E. N. E. Noll, J. Lin, Y. Nakatsuji, R. H. Miller, and P. M. Black, "GM3 as a novel growth regulator for human gliomas," Experimental Neurology, vol. 168, no. 2, pp. 300-309, 2001.

[6] Y. Fujimoto, S. Izumoto, T. Suzuki et al., "Ganglioside GM3 inhibits proliferation and invasion of glioma," Journal of NeuroOncology, vol. 71, no. 2, pp. 99-106, 2005.

[7] F. Guan, K. Handa, and S.-I. Hakomori, "Regulation of epidermal growth factor receptor through interaction of ganglioside GM3 with GlcNAc of N-Linked glycan of the receptor: demonstration in ldlD cells," Neurochemical Research, vol. 36, no. 9, pp. 1645-1653, 2011.

[8] K. Iijima, N. Soga, T. Matsubara, and T. Sato, "Observations of the distribution of GM3 in membrane microdomains by atomic force microscopy," Journal of Colloid and Interface Science, vol. 337, no. 2, pp. 369-374, 2009.

[9] G. Margheri, R. D’Agostino, M. Del Rosso, and S. Trigari, "Fabrication of GM3-enriched sphingomyelin/cholesterol solidsupported lipid membranes on $\mathrm{Au} / \mathrm{SiO}_{2}$ plasmonic substrates," Lipids, vol. 48, no. 7, pp. 739-747, 2013.

[10] G. Margheri, R. D’Agostino, S. Trigari, S. Sottini, and M. Del Rosso, "The $\beta$-subunit of cholera toxin has a high affinity for ganglioside GM1 embedded into solid supported lipid membranes with a lipid raft-like composition," Lipids, vol. 49, no. 2, pp. 203-206, 2014.

[11] F. Margheri, L. Papucci, N. Schiavone et al., "Differential uPAR recruitment in caveolar-lipid rafts by GM1 and GM3 gangliosides regulates endothelial progenitor cells angiogenesis," Journal of Cellular and Molecular Medicine, vol. 19, no. 1, pp. 113123, 2015.

[12] Z. Salamon, H. A. Macleod, and G. Tollin, "Coupled plasmonwaveguide resonators: a new spectroscopic tool for probing proteolipid film structure and properties," Biophysical Journal, vol. 73, no. 5, pp. 2791-2797, 1997.

[13] Y. Mao, R. Tero, Y. Imai, T. Hoshino, and T. Urisu, “The morphology of GM1x/SM0.6-x/Chol0.4 planar bilayers supported on $\mathrm{SiO}_{2}$ surfaces," Chemical Physics Letters, vol. 460, no. 1-3, pp. 289-294, 2008. 
[14] H. Raether, Surface Plasmons on Smooth and Rough Surfaces and on Gratings, Springer, Berlin, Germany, 1980.

[15] P. R. Edwards, P. A. Lowe, and R. J. Leatherbarrow, "Ligand loading at the surface of an optical biosensor and its effect upon the kinetics of protein-protein interactions," Journal of Molecular Recognition, vol. 10, no. 3, pp. 128-134, 1997.

[16] M. A. Yednak and E. G. Bremer, "Preferential binding of the epidermal growth factor receptor to ganglioside GM3-coated plates," Molecular and Chemical Neuropathology, vol. 21, no. 2-3, pp. 369-378, 1994.

[17] A. Abbas, M. J. Linman, and Q. Cheng, "Sensitivity comparison of surface plasmon resonance and plasmon-waveguide resonance biosensors," Sensors and Actuators, B: Chemical, vol. 156, no. 1, pp. 169-175, 2011.

[18] W.-L. Li, R. Jia, M. Liu et al., "Fabrication and characterization of Si nanocrystals synthesized by electron beam evaporation of $\mathrm{Si}$ and $\mathrm{SiO}_{2}$ mixture," Chinese Physics Letters, vol. 26, no. 4, Article ID 046801, 2009.

[19] E. A. Miljan, E. J. Meuillet, B. Mania-Farnell et al., "Interaction of the extracellular domain of the epidermal growth factor receptor with gangliosides," The Journal of Biological Chemistry, vol. 277, no. 12, pp. 10108-10113, 2002.

[20] Y. Haga, S.-I. Hakomori, and K. Hatanaka, "Quantitative analysis of EGFR affinity to immobilized glycolipids by surface plasmon resonance," Carbohydrate Research, vol. 343, no. 18, pp. 3034-3038, 2008. 

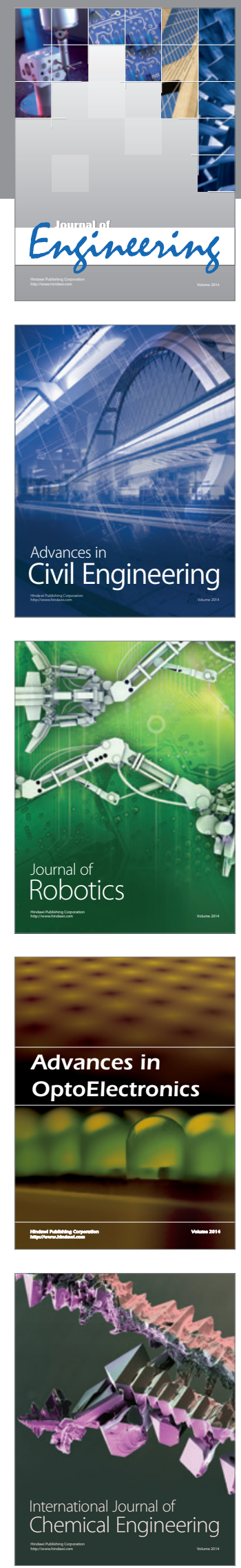

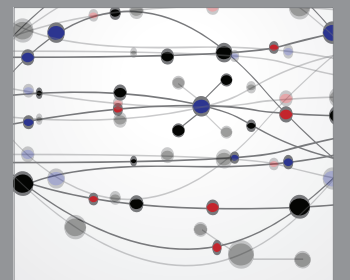

The Scientific World Journal
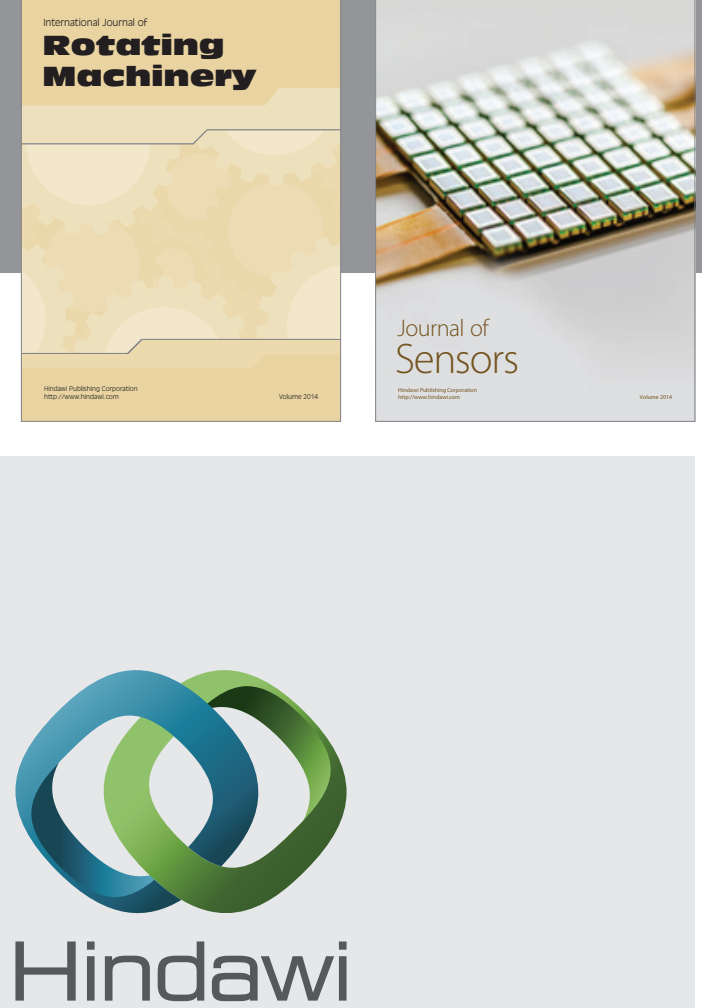

Submit your manuscripts at http://www.hindawi.com
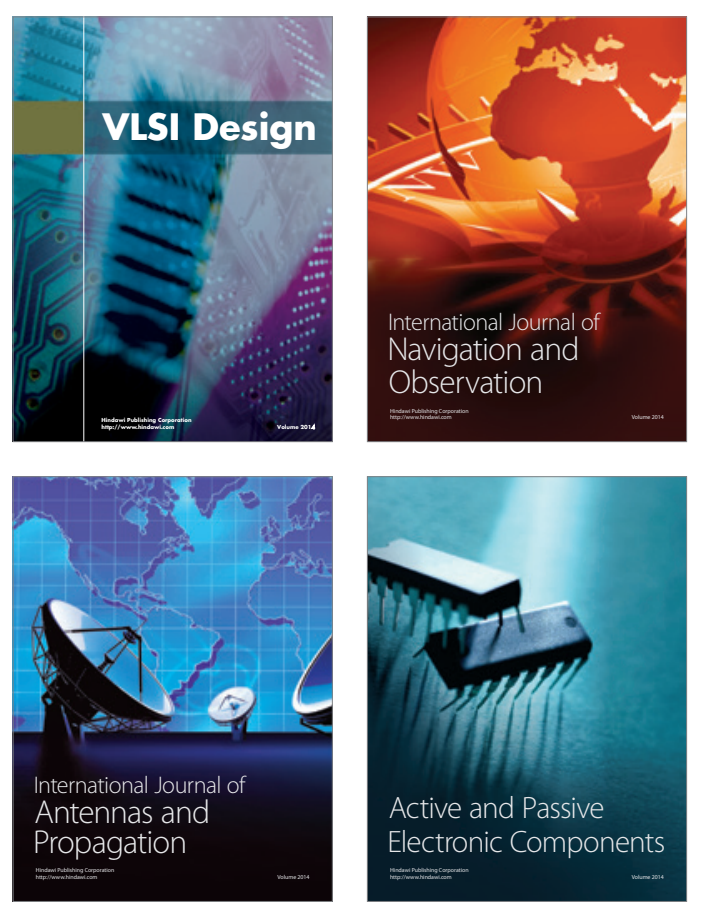
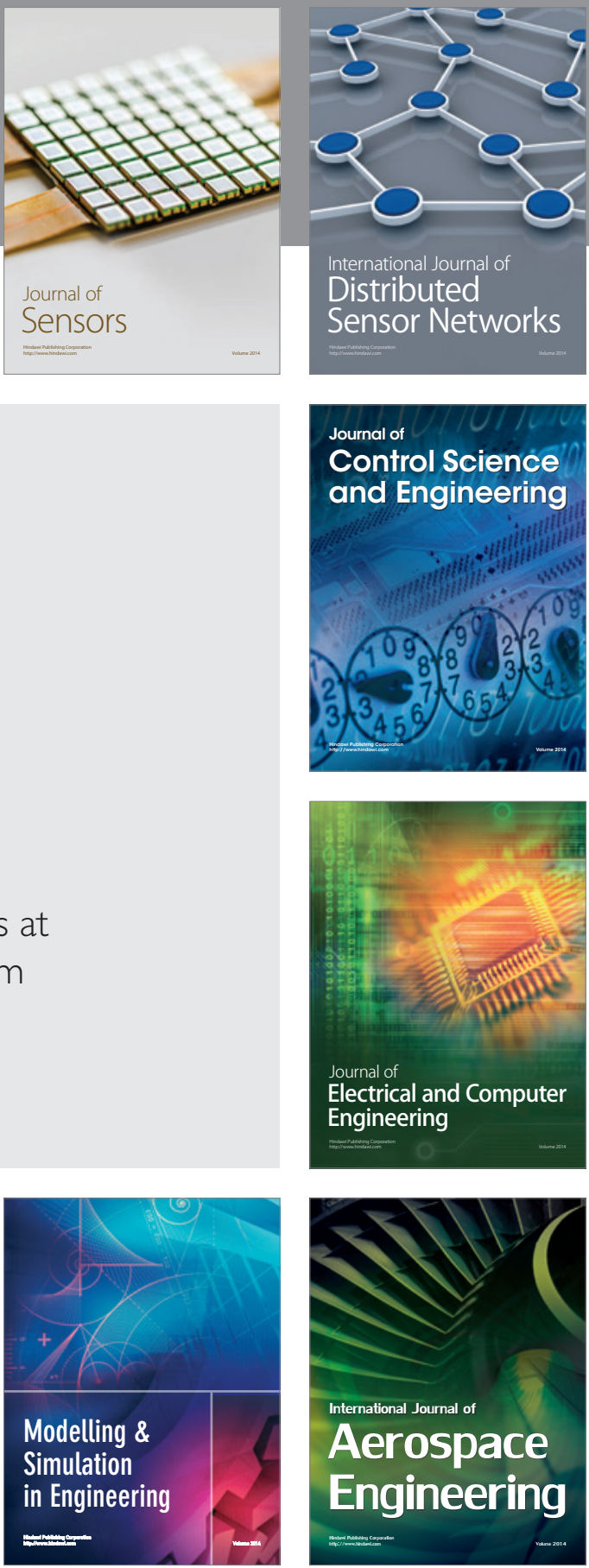

Journal of

Control Science

and Engineering
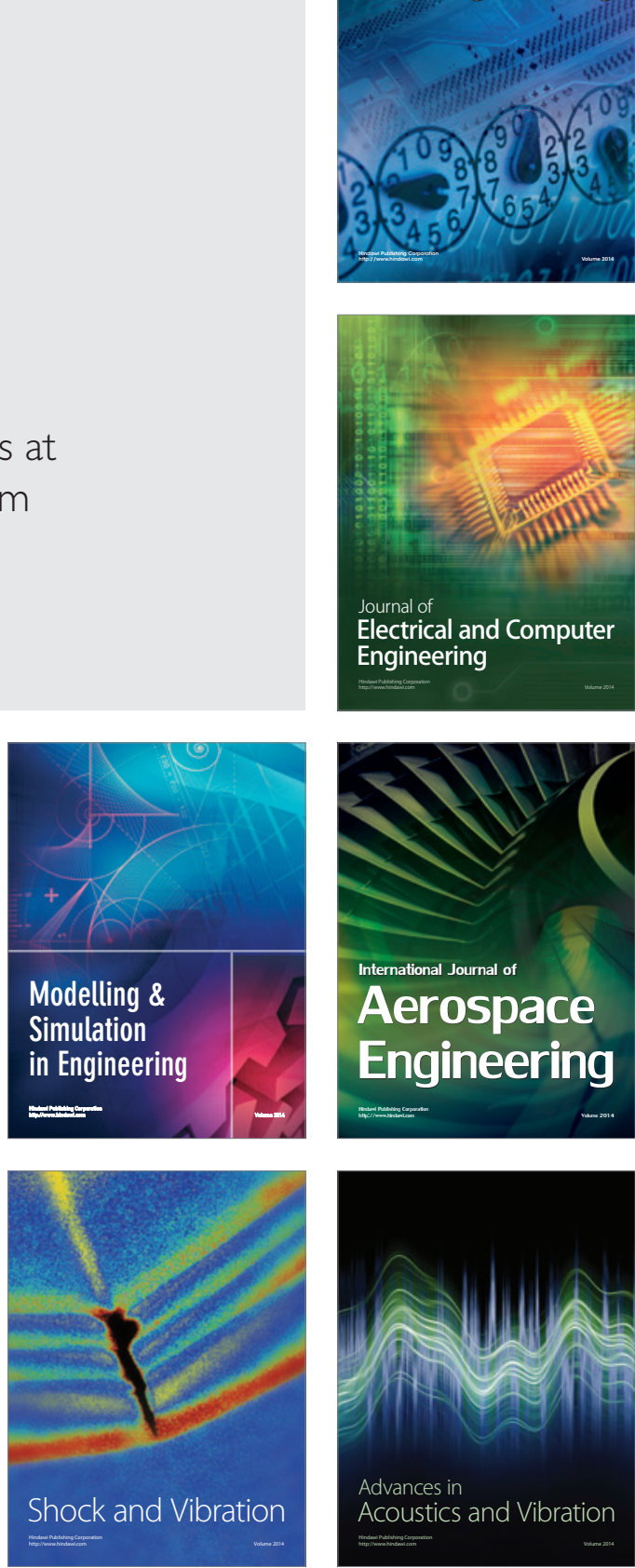